\title{
BMJ Open Public, medical professionals' and patients' preferences for the allocation of donor organs for transplantation: study protocol for discrete choice experiments
}

\author{
Carina Oedingen, ${ }^{1,2}$ Tim Bartling, ${ }^{1,2}$ Christian Krauth ${ }^{1,2}$
}

To cite: Oedingen C, Bartling T, Krauth C. Public, medical professionals' and patients' preferences for the allocation of donor organs for transplantation: study protocol for discrete choice experiments. BMJ Open 2018;8:e026040. doi:10.1136/ bmjopen-2018-026040

- Prepublication history for this paper is available online. To view these files, please visit the journal online (http://dx.doi. org/10.1136/bmjopen-2018026040).

$\mathrm{CO}$ and TB contributed equally.

Received 14 August 2018 Revised 20 August 2018 Accepted 23 August 2018

Check for updates

(c) Author(s) (or their employer(s)) 2018. Re-use permitted under CC BY-NC. No commercial re-use. See rights and permissions. Published by BMJ.

${ }^{1}$ Institute for Epidemiology, Social Medicine and Health Systems Research, Hannover Medical School, Hannover, Germany

${ }^{2}$ Center for Health Economics Research Hannover (CHERH), Hannover, Germany

\section{Correspondence to} Carina Oedingen; oedingen.carina@mh-hannover. de

\section{ABSTRACT}

Introduction Organ transplantation is the treatment of choice for patients with severe organ failure. Nevertheless, donor organs are a scarce resource resulting in a large mismatch between supply and demand. Therefore, priority-setting leads to the dilemma of how these scarce organs should be allocated and who should be considered eligible to receive a suitable organ. In order to improve the supply-demand mismatch in transplantation medicine, this study explores preferences of different stakeholders (general public, medical professionals and patients) for the allocation of donor organs for transplantation in Germany. The aims are (1) to determine criteria and preferences, which are relevant for the allocation of scarce donor organs and (2) to compare the results between the three target groups to derive strategies for health policy. Methods and analysis We outline the study protocol for discrete choice experiments, where respondents are presented with different choices including attributes with varied attribute levels. They were asked to choose between these choice sets. First, systematic reviews will be conducted to identify the state of art. Subsequently, focus group discussions with the public and patients as well as expert interviews with medical professionals will follow to establish the attributes that are going to be included in the experiments and to verify the results of the systematic reviews. Using this qualitative exploratory work, discrete choice studies will be designed to quantitatively assess preferences. We will use a D-efficient fractional factorial design to survey a total sample of 600 respondents according to the public, medical professionals and patients each. Multinomial conditional logit model and latent class model will be analysed to estimate the final results. Ethics and dissemination This study has received Ethics Approval from the Hannover Medical School Human Ethics Committee (Vote number: 7921_B0_K_2018). Findings will be disseminated through conference presentations, workshops with stakeholders and peer-reviewed journal articles.

\section{INTRODUCTION}

Since its beginning, transplantation medicine worldwide is faced with the challenge of a mismatch in supply and demand for donor organs, forcing stakeholders to make decisions on how these scarce resources should

\section{Strengths and limitations of this study}

- To our knowledge, this study will be the first to measure and quantify stated preferences (discrete choice experiments) for deceased donor organ allocation considering three different stakeholder groups: the general public, medical professionals and patients.

- This approach allows a complete overview and comparison between different stakeholders, since discrete choice experiments provide a better understanding of preferences than instruments which use surveys measuring preferences directly.

- The attributes and levels will be selected based on findings of preliminary work such as systematic reviews as well as qualitative research, in order to identify which aspects of organ allocation are most important and to provide a realistic context.

- The results will contribute a better understanding of preferences and an improvement of the organ allocation policy, in order to increase the number of available organs.

- Given a scenario, the design of discrete choice experiments demands participants to make a decision on what they might think, while not assessing actual decisions, which is a limitation of the methodology.

be allocated and who should be considered to receive an available organ. ${ }^{1}$ This decision-making and priority-setting is an ethical dilemma, because for most patients with severe organ failure, a new healthy organ is the last chance to live. However, organ allocation procedures need to be seen as a complex case, in which multiple factors must be considered. ${ }^{2}$ To date, allocation of deceased donor organs is based on criteria such as time on the waiting list, medical urgency, chance of success or paediatric status. ${ }^{3}$ In Germany, for example, all organ allocation decisions are recommended to explicitly consider medical urgency and transplantation success according to the 1997 German organ transplantation act. ${ }^{4}$ Yet, these 
criteria are conflicting since success rate usually decreases if urgency increases. ${ }^{5}$

How to allocate organs should be seen as a societal task, since selecting a specific patient always comes with deselecting other patients, which might not have the chance to receive another organ in the future. Therefore, the decision-making as well as priority-setting is dependent from different stakeholders: general public (they are the potential suppliers of organs and determine how many organs are available for transplantation), medical professionals (they are the healthcare providers responsible for the patients) as well as patients (they are the recipients of the new organs).

\section{Public preferences of organ allocation}

It is important to involve public preferences in the organ allocation decisions, since organ donation needs to be seen as a public resource: Supply and demand for organs is highly dependent on the amount of people donating and people needing, respectively. To date, most studies and overviews focused on organ donation rather than organ allocation. ${ }^{6-12}$ A systematic review by Tong et $a l^{13}$ ascertained seven main themes underlying public preferences for organ allocation: 'maximum benefit' (survival and quality of life), 'social valuation' (with dependants, employed or respected occupation), 'moral deservingness' (registered donor, healthy lifestyle), 'prejudice' (citizen, ethnicity), 'fair innings' (younger age, first transplant), 'first come, first served' (longer waiting time) as well as 'medical urgency' (more severe illness, closer to death). All relevant themes occur simultaneously, resulting in rather conflicting preferences between individuals, whereby an overall trade-off between different criteria was not identifiable. However, most of the single studies ascertain public preferences with direct questionnaires, showing that the public wants to prioritise those with the most benefit, while also considering those with the highest urgency or longest waiting time..$^{14-18}$

\section{Medical professionals' preferences of organ allocation}

Medical professionals have a very unique role in the process of organ allocation. They are the gatekeeper for patients to organ allocation, starting with informing the patients about the procedure, initiating organisational procedures when perceiving their patients as in need of an organ and taking the necessary medical steps for their transplantation. ${ }^{19}$ So far, many studies on this topic identified that some candidates are seen as more deserving to receive an organ than others, whereas patients with suicidal tendencies, HIV, alcoholism or a history of substance abuse are seen as undeserving. ${ }^{20}$ Furthermore, a lot of medical factors are seen as clear contraindications to transplantation, such as diabetes, active systematic lupus erythematosus or inoperable coronary artery disease. ${ }^{19}$ Studies assessing the preferences of medical professionals regarding organ allocation are often asking either for agreement/disagreement towards specific statements ${ }^{21} 22$ or for an opinion of whether a hypothetical patient is eligible for transplantation. ${ }^{23}$ Comparing patients and deciding for one, or ranking criteria regarding their importance in allocation eligibility is rarely done. Also, qualitative interviews have usually been explorative. ${ }^{24} 25$

\section{Patients' preferences of organ allocation}

The patients act as a counterpart to the medical professionals in this case. While medical professionals act as advocates for their patients and should decide in their best interest, ${ }^{26}$ in practice this relationship might be different. Patients are affected by their own medical condition and have preferences shaped by the extreme situation they are in. A common design for studies involving patients is comparing two hypothetical candidates and investigating the impact of the trade-offs offered on the patients' decisions. This is often done via scenario-based questionnaires. ${ }^{27-30}$ Additionally, patients are often asked to rank attributes according to their personal preferences or according to a group consensus. ${ }^{31} 32$ While some personal factors, such as having children or other dependants, are seen as highly important, ${ }^{29} 3032$ other personal factors, such as being a donor themselves, are not perceived as relevant. ${ }^{3133}$ They intuitively understand that scarce resources need to be allocated by highest efficiency, ${ }^{33}$ and also agree with prioritising patients by longer waiting time. $^{27} 31$ To our knowledge, no systematic reviews have assessed the perspectives of patients regarding prioritising candidates for organ allocation so far.

\section{AIMS}

This paper describes the study protocol of the 'Präferenzen in der Organallokation' (POL) (Preferences in Organ Allocation) study. This study elicits how the three target groups of public, medical professionals and patients in Germany want to allocate donor organs and what aspects of organ allocation influence their preferences. The specific objectives are:

1. Extract criteria and preferences on how donor organs should be allocated.

2. Assess the extent to which preferences vary between the three target groups.

3 . Determine the relative importance of different attributes that go beyond transplantation success and medical urgency.

This study is using liver transplantations as the example of allocation decisions. Furthermore, we compare the public preferences with current ethical principles of distributive justice. We expect that the results allow a better understanding of preferences between different stakeholders and contribute to influence the organ allocation policy.

\section{METHODS AND ANALYSIS}

\section{Overview of approach and method: discrete choice} experiment development

The POL study will use both quantitative and qualitative methods including systematic reviews, focus group discussions, expert interviews and discrete choice experiments 
(DCEs) to investigate preferences from the public, medical professionals and patients regarding allocation of donor organs for transplantation. It should be noted that qualitative work is required as a part of DCE development in order to identify relevant attributes and levels for the choice design. ${ }^{34}$

A DCE is a common method to elicit stated preferences, which has been increasingly used in health economics regarding among others to assess health or healthcare services. ${ }^{35}$ It asks its respondents to imagine scenarios, designed to mimic real life and make a choice on the basis of their own preferences between different alternatives in each scenario. The method is based on Lancaster's theory ${ }^{36}$ that (1) the utility of goods and services can be described or defined by different key characteristics or factors (ie, attributes) that characterise the good or service and that (2) each attribute varied systematically with different specifications (ie, attribute levels). In a DCE, a good or service is described with relevant attributes based on changing combinations of attribute levels. Respondents are asked to choose between a number of hypothetical alternatives (ie, choice sets) the option that is most preferred. It is assumed that respondents take into account all information provided and select the option that has the highest value or utility for them. From these choices, a mathematical model can be constructed which describes the relative importance (ie, trade-off) of the attributes. Therefore, DCE studies allow determining which attributes are driving individual's preferences, which trade-offs respondents are willing to accept and how changes in attributes and attribute levels affect the respective preferences. ${ }^{36}$ Furthermore, DCEs are based on the random utility theory, ${ }^{37}$ which means, in contrast to the classic consumer theory, that individual choice behaviour is rather probabilistic than deterministic. This implies that the utility of a good or service can be divided into an explicable, systematic element as well as a non-explicable, random element. The latter, for example, can be due to unobserved preferences or measurement errors. ${ }^{38} 39$

According to the aims of the POL study, three substudies with the perspective of the public, medical professionals and patients will be conducted. The same methods and processes will be used for each study, as described in the five steps below. The study will follow the International Society for Pharmacoeconomics and Outcomes Research best practices guidelines for Good Research Practices for Conjoint Analysis Task Force. ${ }^{40}$ An overview of the DCE development process is shown in figure 1. We are currently in the first stage (completed systematic review) of identifying attributes of organ allocation.

\section{Stage 1: systematic review}

Two systematic reviews were conducted to identify which aspects of organ allocation are most important for the target groups. Irrespective of study design, all quantitative and qualitative studies evaluating preferences and perspectives regarding the allocation of donor organs and listed from 1 January 2000 to 1 November 2017 were searched in three databases without language restriction: PubMed, Web of Science Core Collection and EBSCO Biomedical Reference Collection: Corporate Edition. Only studies from the year 2000 onwards were included in order to represent up-to-date preferences and perspectives as well as to consider changes in transplantation medicine. In addition, we checked reference lists from retrieved literature for relevant publications. Two researchers independently screened studies in a three-step process. First, titles were screened to identify potentially relevant studies. Subsequently, the abstracts of the included titles were screened in order to assess the studies still relevant. These were then read in full for more detail. The screening was conducted in a sensitive approach. Disagreements were discussed until consensus was reached and the help of a third reviewer was consulted in cases where consensus could not be reached.

For full transparency, we will publish the results of both systematic reviews.

\section{Stage 2: qualitative research}

Focus group discussions and expert interviews will be conducted both to establish the attributes to be included in the DCEs and to verify the results of the systematic reviews. Overall, three to four focus groups will be performed with the involvement of public and patients $(n=8-12)$. The final number of focus groups realised will be based on the point of data saturation. The public will be recruited via Hannover Medical School event series 'Patientenuniversität' ('patient university', an independent health education institution to increase health literacy in the general population) as well as via newspaper advertising. These participants will be sorted in one group with people who do not have any information/ experiences about organ transplantation and allocation, one who does and a third, mixed group. Patients with liver failure will be recruited from Hannover Medical School and support groups. Participants will be purposively sampled to ensure a balance of numbers between male and female, age groups and a variety of cultural backgrounds, when possible. Each focus group will have three phases: (1) preliminary questions about general thoughts and attitudes to organ transplantation and allocation, (2) a group discussion on what factors should be considered in organ allocation and how people should be prioritised to receive organs and (3) a ranking exercise of the factors identified from the group discussion. The ranking exercise will be conducted using a modified nominal group technique. ${ }^{41-43}$ Furthermore, we will conduct four to six expert interviews with medical professionals, (physicians and surgeons involved in the transplantation process, transplantation commissioned) recruited via Hannover Medical School as well as national and international congresses and conferences. The final number realised is also dependent on achieved data saturation.

For all target groups, people who are interested in participating in the focus group discussions or face-toface interviews are asked to respond to the study advertisements by contacting the research team through email 


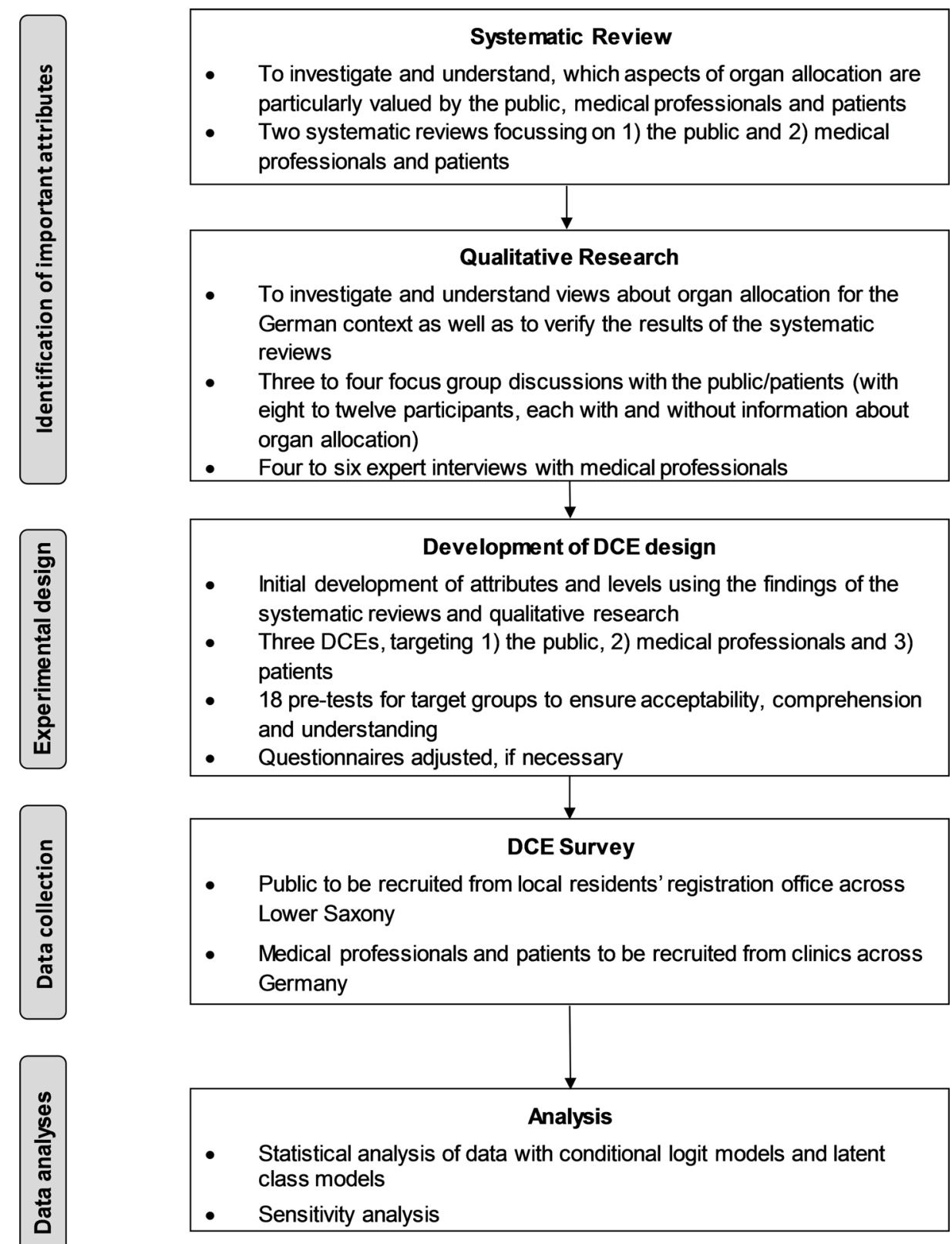

Figure 1 Development of the DCEs. DCEs, discrete choice experiments.

or telephone for more information. Following contact, we will provide additional information on the intention to ensure participants have adequate knowledge and understand what their participation would involve. All participants must be aged 18 years or older, able to speak German and with the ability to provide consent. If they meet these inclusion criteria, participants will be asked to provide an email or postal address to send an invitation including the participant information sheet and consent form. All sessions will be moderated by the research team and will be digitally audio recorded and later transcribed in full without any identifying information. The focus group discussions will last approximately 1.5-2 hours while the expert interviews will last $30-45 \mathrm{~min}$. Transcripts will be entered into MAXQDA (VERBI Software GmbH V.18.0.8) and reviewed line by line by the research team.
A preliminary coding system will be developed and the data will be analysed for emergent themes using content analysis according to Mayring. ${ }^{44}$

\section{Stage 3: development of DCE design}

Following the preliminary work in stages 1 and 2, a design for the DCE studies will be created when the attributes and attribute levels have been decided on. The final list of attributes and attribute levels will be selected from current recommendations ${ }^{40}$ by (1) considering any possible important attribute for the organ allocation and (2) including those who could be realistically described in choice scenarios without creating dominant options. A process of selecting and specifying these attributes and attribute levels will be followed. 
In an unlabelled DCE, the inclusion of the exact number of attributes and attribute levels is $\mathrm{L}^{\mathrm{A}}$ (if equal in all attributes), where $\mathrm{L}$ is the number of attribute levels and $\mathrm{A}$ the number of attributes. Because it is not feasible to confront all respondents with all possible combinations of attribute levels due to time frame and fatigue constraints, each respondent will be presented with a subset of all possible choice sets, a so-called fractional factorial design. This practical purpose reduces the number of choices used in the experiment while maximising the statistical efficiency (ie, precision) of the design. Therefore, a design maximised for the multinomial logit model based on D-efficient fractional factorial experimental criteria will be used to generate a certain number of pairwise choice sets using the design software SAS OnDemand for Academics (SAS V.9.4). ${ }^{39} 4546$ We will generate a design that can estimate the organ allocation criteria effects as well as potential interactions between different criteria effects. Previous research suggests, based on rules of thumb, that respondents can efficiently handle approximately 10 choice sets at one time if they do not have any cognitive impairment ${ }^{47}$ and that the number of included attributes should be limited to maximum eight each with two to four levels. ${ }^{39}{ }^{40}$ It should be noted that the design has to enable a realistic and comprehensive image resulting in little descriptions of the used attributes, which were supported visually. Consequently, the final design will be divided into several blocks (ie, versions) of the survey with 10 choice sets per block for all three target groups. Blocking is an accepted statistical technique in a DCE design and will be used to ensure balance among differing attribute levels. ${ }^{45}$ Every version of the survey will be randomly allocated to each respondent by the software used to design the survey. An example of a DCE choice task is shown in figure 2.

The DCE questionnaire will be tested for face and theoretical validity. Pilot data will be collected on approximately 18 respondents from the public, medical professionals and patients. Face validity will be explored refining the phrasing and comprehension of the respondents and asking at the end of the survey what they thought could be improved about the survey. Whereas theoretical validity will be tested through sign and significance of the parameter estimates to ensure that they are conform to priori expectations, especially without dominant attributes. Additionally, two choice sets will also be included to serve as consistency and reliability checks. A consistency check is a theoretically dominant choice set which is used to test the rationality of the respondents and a reliability check is simply a repetition of a choice set from the experimental design to somewhere later. ${ }^{48} 49$

\section{Stage 4: DCE survey}

The questionnaires will be structured into an introduction, an explanation of the context of the survey, an example of the choice set with descriptions of all used attributes and attribute levels, a suggestion on respondents' time and the importance of their participation and confidentiality. In addition to the DCE questions, information on socioeconomic and sociodemographic characteristics as well as attitude to organ transplantation and allocation via Likert scales will be collected for each survey. The questionnaires will also include questions on a Likert scale, which will allow an evaluation of the level difficulty of the choice sets and how confident the respondents were with their decisions.

The DCE survey will be conducted using a paper-andpencil questionnaire for all three target groups with an option for an additional online survey for the public. For the public survey, we will use a quota sampling based on age and sex to recruit a sample broadly representative of the German public. Respondents will be recruited from local residents' registration office across Lower Saxony. Medical professionals and patients will be recruited through clinics across Germany. Inclusion criteria are the same as with the qualitative research.

To date, the sample size calculations for DCE studies have not been completely developed. Therefore, a final power calculation cannot be performed. However, there is ambiguity about the relevance of sample size calculation in DCEs because the sampling theory rather is designed to minimise errors in the choice proportions of the alternatives than to calculate the minimum sample size to generate reliability of the estimated effects.

Figure 2 Example of a DCE choice set. DCE, discrete choice experiment. 
Therefore, the final sample size is dependent on the design characteristics of the DCE, whereby the number of attributes, the attribute levels, the number of choice sets, the number of alternatives in each choice set and the size and direction of prior parameters obtained from the pilot study has been decided before sample size for DCEs can be calculated. ${ }^{50}$ However, previous studies using DCE design have typically shown that 300-400 respondents may be sufficient for statistical analysis. ${ }^{35}$ Johnson and Orme ${ }^{52} 53$ propose their calculation for a minimal sample size: $\frac{\mathrm{nta}}{c} \geq 500$, where $\mathrm{n}$ is the minimal sample size, t the number of choice sets, a the number of alternatives in each choice set and $c$ the highest number of level in attributes included. It should be noted that for robust results the formal should conclude to a minimum of 500 . More desirable should be a final value of $1000 .^{54}$ To ensure that the respondents are broadly generalisable to the German adult population and that we are able to explore interactions between different attributes as well as to analyse subgroups, we plan with a sample size of approximately 200 respondents in each of the public, medical professionals and patients resulting in a total of 600 respondents.

\section{Stage 5: analysis}

Data obtained from the paper-and-pencil questionnaires will be entered directly by one of the study researchers into Microsoft Excel (Microsoft Corporation V.2010), and will be checked by a second researcher. Data will be imported into Stata (StataCorp V.15) and frequency distributions will be used to check for and exclude outliers. All three target groups will be analysed separately and afterwards will be checked against each other. First, descriptive statistics will be calculated for respondent samples. Second, data to the DCE will be analysed using multinomial conditional logit model (MCLM) and latent class models (LCM). An MCLM is the most basic choice model used as a comparison when estimating more complex models ${ }^{51}$ and estimates average preferences as fixed values while an LCM allows to identify correlations in responses and to assess preference heterogeneity. Therefore, LCMs group respondents into classes which have similar preferences to identify respondents characteristics associated with particular groups. Finally, sensitivity analyses will be performed to determine the stability of the parameter estimates.

Model results will be expressed as parameter estimates $(\beta)$, odds of one choice instead of another choice and their $95 \%$ CIs as well as $p$ values. The results of this study will inform policy about public, medical professionals' and patients' preferences for the allocation of donor organs for transplantation by highlighting factors, which are most important in the allocation as well as factors, which differentiate between the three target groups.

\section{Patient and public involvement}

We are planning to involve patient support groups in the recruitment process. Patients and public were neither involved in the development of the research questions or the outcome measures, nor in the study design and will not be used for conducting the research except of their role as respondents. Every participant (patients, public as well as medical professionals) will be informed that they can request the study results in written form at the end of the project.

\section{ETHICS AND DISSEMINATION}

Confidentiality and anonymity of the obtained data will be strictly guaranteed. Digital recording of the focus groups and expert interviews will only be performed after written informed consent is given from the participants. Participants will not be identifiable in any transcripts. It will be stated clearly that all participants have the right to withdraw from the research at any point in time without any disadvantages. For the DCE survey, no formal written consent will be needed, as consent will be implied by completion and sending of the questionnaire. Additionally, written consent is not possible if the public will be surveyed via an online platform. Overall, data protection is guaranteed and secured according to the internal data protection office from the Hannover Medical School.

Submission of abstracts to various conferences and publication in peer-reviewed medicine as well as public health and social science journals is to follow. Results will also be communicated to the funding body by way of an annual report. To develop strategies for wider dissemination of the findings, we will organise workshops at the end of the project with decision-makers in the field of health policy as well as stakeholders. By supplying a better understanding of current preferences in the public, medical professionals and patients, our study will highlight options to allow for more transparent and equitable policies to organ allocation and in the consequence to increase the willingness to donate organs.

Contributors CK wrote the grant application. $\mathrm{CO}$ wrote the first draft of the protocol. TB supported $\mathrm{CO}$ in writing the protocol. CO, TB and CK contributed to the drafting and editing of the protocol and approved the final version.

Funding This work was supported by the German Federal Ministry of Education and Research grant number 01EH1603B.

Competing interests None declared.

Patient consent Not required.

Ethics approval The POL study has been approved by the Hannover Medical School, Human Research Ethics Committee (Vote number: 7921_B0_K_2018).

Provenance and peer review Not commissioned; peer reviewed for ethical and funding approval prior to submission.

Open access This is an open access article distributed in accordance with the Creative Commons Attribution Non Commercial (CC BY-NC 4.0) license, which permits others to distribute, remix, adapt, build upon this work non-commercially, and license their derivative works on different terms, provided the original work is properly cited, appropriate credit is given, any changes made indicated, and the use is non-commercial. See: http://creativecommons.org/licenses/by-nc/4.0/.

\section{REFERENCES}

1. Eurotransplant International Foundation. Annual Report 2016. Leiden: Eurotransplant Foundation, 2016. 
2. Stegall MD. The development of kidney allocation policy. Am J Kidney Dis 2005;46:974-5.

3. Leichtman AB, McCullough KP, Wolfe RA. Improving the allocation system for deceased-donor kidneys. N Engl J Med 2011;364:1287-9.

4. Gesetz über die Spende, Entnahme und Übertragung von Organen und Geweben (Transplantationsgesetz - TPG) in der Fassung der Bekanntmachung vom 4. September 2007 (BGBI. I S. 2206), das zuletzt durch Artikel 2 des Gesetzes vom 18. Juli 2017 (BGBI. I S. 2757) geändert worden ist [Act on organ and tissue donation, removal and transplantation (German Transplant Act (TPG)].

5. Bobbert M, Ganten TM. Liver allocation: urgency of need or prospect of success? Ethical considerations. Clin Transplant 2013;27:34-9.

6. Broumand M, Parsapoor A, Asghari F. Public opinion of organ donation: a survey in Iran. Clin Transplant 2012;26:E500-4.

7. Chandler JA, Burkell JA, Shemie SD. Priority in organ allocation to previously registered donors: public perceptions of the fairness and effectiveness of priority systems. Prog Transplant 2012;22:413-22.

8. Howard K, Jan S, Rose JM, et al. Preferences for policy options for deceased organ donation for transplantation: a discrete choice experiment. Transplantation 2016;100:1136-48.

9. Irving MJ, Jan S, Tong A, et al. What factors influence people's decisions to register for organ donation? The results of a nominal group study. Transpl Int 2014;27:617-24.

10. Li AT, Wong G, Irving M, et al. Community-based interventions and individuals' willingness to be a deceased organ donor: systematic review and meta-analysis. Transplantation 2015;99:2634-43.

11. Moorlock G, Ives J, Bramhall S, et al. Should we reject donated organs on moral grounds or permit allocation using non-medical criteria?: a qualitative study. Bioethics 2016;30:282-92.

12. Morgan SE, Harrison TR, Afifi WA, et al. In their own words: the reasons why people will (not) sign an organ donor card. Health Commun 2008;23:23-33.

13. Tong A, Howard K, Jan S, et al. Community preferences for the allocation of solid organs for transplantation: a systematic review. Transplantation 2010;89:796-805.

14. Asghari F, Broumand M, Heidari A. Public preferences for donor kidney allocation: a study in Iran. Clin Transplant 2013;27: 718-23.

15. Balwani MR, Gumber MR, Shah PR, et al. Attitude and awareness towards organ donation in western India. Ren Fail 2015;37:582-8.

16. Howard K, Jan S, Rose JM, et al. Community preferences for the allocation of donor organs for transplantation: a discrete choice study. Transplantation 2015;99:560-7.

17. Ratcliffe J. Public preferences for the allocation of donor liver grafts for transplantation. Health Econ 2000;9:137-48.

18. Stahl JE, Tramontano AC, Swan JS, et al. Balancing urgency, age and quality of life in organ allocation decisions--what would you do? A survey. J Med Ethics 2008;34:109-15.

19. Tong A, Hanson CS, Chapman JR, et al. The preferences and perspectives of nephrologists on patients' access to kidney transplantation: a systematic review. Transplantation 2014;98:682-91.

20. Tong A, Jan S, Wong G, et al. Rationing scarce organs for transplantation: healthcare provider perspectives on wait-listing and organ allocation. Clin Transplant 2013;27:60-71.

21. Cass $\mathrm{A}$, Cunningham J, Anderson $\mathrm{K}$, et al. Decision-making about suitability for kidney transplantation: results of a national survey of Australian nephrologists. Nephrology 2007;12:299-304.

22. Almeida N, Almeida RF, Almeida K, et al. Attitude of medical professionals regarding controversial issues in kidney donation/ transplantation. Indian J Nephrol 2016;26:393-7.

23. Thamer M, Hwang W, Fink NE, et al. U.S. nephrologists' attitudes towards renal transplantation: results from a national survey. Transplantation 2001;71:281-8.

24. Tong A, Howard K, Wong G, et al. Nephrologists' perspectives on waitlisting and allocation of deceased donor kidneys for transplant. Am J Kidney Dis 2011;58:704-16.

25. Omar F, Tinghög G, Carlsson $P$, et al. Priority setting in kidney transplantation: a qualitative study evaluating Swedish practices. Scand J Public Health 2013;41:206-15.

26. World Medical Association (WMA). Medical Ethics Manual. 3rd edn. Ferney-Voltaire Cedex: WMA, 2015.

27. Geddes CC, Rodger RS, Smith C, et al. Allocation of deceased donor kidneys for transplantation: opinions of patients with CKD. Am $J$ Kidney Dis 2005;46:949-56.

28. Umgelter KS, Tobiasch M, Anetsberger A, et al. Donor organ distribution according to urgency of need or outcome maximization in liver transplantation. A questionnaire survey among patients and medical staff. Transpl Int 2015;28:448-54.
29. Clark MD, Gumber A, Leech D, et al. Prioritising patients for renal transplantation? Analysis of patient preferences for kidney allocation according to ethnicity and gender. Diversity in Health and Care 2009;6:181-91.

30. Clark MD, Leech D, Gumber A, et al. Who should be prioritized for renal transplantation?: analysis of key stakeholder preferences using discrete choice experiments. BMC Nephrol 2012;13:152.

31. Tong A, Jan S, Wong G, et al. Patient preferences for the allocation of deceased donor kidneys for transplantation: a mixed methods study. BMC Nephrol 2012;13:18.

32. Gibbons A, Cinnirella M, Bayfield J, et al. Patient preferences, knowledge and beliefs about kidney allocation: qualitative findings from the UK-wide ATTOM programme. BMJ Open 2017;7:e013896.

33. Lawrence C, Sharma S, Da Silva-Gane M, et al. Exploring the views of patients not on the transplant waiting list: a qualitative study. $J$ Ren Care 2013;39:118-24

34. Coast J, Al-Janabi $\mathrm{H}$, Sutton $\mathrm{EJ}$, et al. Using qualitative methods for attribute development for discrete choice experiments: issues and recommendations. Health Econ 2012;21:730-41.

35. de Bekker-Grob EW, Ryan M, Gerard K. Discrete choice experiments in health economics: a review of the literature. Health Econ 2012;21:145-72.

36. Lancaster KJ. A new approach to consumer theory. J Polit Econ 1966;74:132-57.

37. McFadden D. Conditional logit analysis of qualitative choice behavior. In: Zarembka P, ed. Frontiers in econometrics. New York: Academic Press, 1974:105-42.

38. Lancsar E, Fiebig DG, Hole AR. Discrete choice experiments: a guide to model specification, estimation and software. Pharmacoeconomics 2017;35:697-716.

39. Lancsar E, Louviere J. Conducting discrete choice experiments to inform healthcare decision making: a user's guide. Pharmacoeconomics 2008:26:661-77.

40. Bridges JF, Hauber AB, Marshall D, et al. Conjoint analysis applications in health--a checklist: a report of the ISPOR good research practices for conjoint analysis task force. Value Health 2011;14:403-13.

41. McMillan SS, King M, Tully MP. How to use the nominal group and Delphi techniques. Int J Clin Pharm 2016;38:655-62.

42. Hiligsmann M, van Durme C, Geusens P, et al. Nominal group technique to select attributes for discrete choice experiments: an example for drug treatment choice in osteoporosis. Patient Prefer Adherence 2013;7:133-9.

43. Harvey N, Holmes CA. Nominal group technique: an effective method for obtaining group consensus. Int $J$ Nurs Pract 2012;18:188-94.

44. Mayring P. Qualitative content analysis: theoretical foundation, basic procedures and software solution. Weinheim: Beltz, 2014.

45. Reed Johnson F, Lancsar E, Marshall D, et al. Constructing experimental designs for discrete-choice experiments: report of the ISPOR conjoint analysis experimental design good research practices task force. Value Health 2013;16:3-13.

46. Bliemer MCJ, Rose JM, Hensher DA. Efficient stated choice experiments for estimating nested logit models. Trans Res Part $B$ Method 2009;43:19-35.

47. Netten A, Burge P, Malley J, et al. Outcomes of social care for adults: developing a preference-weighted measure. Health Technol Assess 2012;16:1-166

48. Ryan M, Major K, Skåtun D. Using discrete choice experiments to go beyond clinical outcomes when evaluating clinical practice. $J$ Eval Clin Pract 2005;11:328-38.

49. Carlsson F, Mørkbak MR, Olsen SB. The first time is the hardest: a test of ordering effects in choice experiments. $J$ Choice Model 2012:5:19-37.

50. de Bekker-Grob EW, Donkers B, Jonker MF, et al. Sample size requirements for discrete-choice experiments in healthcare: a practical guide. Patient 2015;8:373-84.

51. Henscher DA, Greene WH. Applied choice analysis: a primer. Cambridge: Cambridge University Press, 2005

52. Orme B. Sample size issues for conjoint analysis studies. Sequim: Sawtooth Software Technical Paper, 1998.

53. Johnson R, Orme B. Getting the most from CBC. Sequim: Sawtooth Software Research Paper Series, Sawtooth Software, 2003.

54. Mühlbacher A, Johnson FR. Choice experiments to quantify preferences for health and healthcare: state of the practice. Appl Health Econ Health Policy 2016;14:253-66. 